\title{
Remaining Useful Life Prediction of Rolling Element Bearings based on Unscented Kalman Filter
}

\author{
Junyu Qi ${ }^{1,2}$, Alexadre Mauricio ${ }^{1,2}$, Mathieu Sarrazin ${ }^{3}$, Karl Janssens ${ }^{3}$, and \\ Konstantinos Gryllias ${ }^{1,2}$ \\ 1 Division PMA, Department of Mechanical Engineering, Faculty of Engineering \\ Science, KU Leuven, Celestijnenlaan 300, BOX 2420, 3001 Leuven, Belgium, \\ junyu.qi@kuleuven.be, \\ 2 Dynamics of Mechanical and Mechatronic Systems, Flanders Make, Belgium \\ 3 Siemens Industry Software NV, Leuven, Belgium
}

\begin{abstract}
A data-driven methodology is considered in this paper focusing towards the Remaining Useful Life (RUL) prediction. Firstly, diagnostic features are extracted from training data and an analytical function that best approximates the evolution of the fault is determined and used to learn the parameters of an Unscented Kalman Filter (UKF). UKF is based on the recursive estimation of the Classic Kalman Filter (CKF) and the Unscented Transform, presenting advantages over the Extended Kalman Filter (EKF) for high non-linear systems. The learned UKF is further applied on testing data in order to predict the RUL under different operating conditions. The influence of the starting point of the prediction is analyzed and a method for the automated parameter tuning of the Kalman Filter is considered. In the end, the result is evaluated and compared to CKF and EKF on experimental data based on dedicated performance metrics.
\end{abstract}

Keywords: Prognostics, Remaining Useful Life, Bearing degradation, Kalman Filter, Parameter tuning

\section{Introduction}

In the era of Industry 4.0, the demand for condition monitoring (CM) of rotating machinery becomes continuously ever more significant and important. The traditional CM pyramid consists of five sub-parts [1]: the reactive maintenance, the preventative maintenance, the condition-based maintenance $(\mathrm{CBM})$, the predictive maintenance and the reliability-centred maintenance. CBM dominates currently in the research and industry, extracting the representative information from the measured signal (vibration, acoustics, oil, etc.) for fault detection and diagnosis. However, prognostics and health management (PHM) nowadays attract unprecedented attention, as it is able to facilitate pre-warning of failure, avoidance of abrupt breakdown, reduction of life circle cost and optimization in 
geometry and materials. The considerable economic value reaches up to $12 \%$ less scheduled repair, $30 \%$ reduction of overall maintenance cost and $70 \%$ elimination of equipment failure [2].

The key point in the PHM lies in the prognostics and the RUL prediction from historical data. RUL refers to the remaining functional time from the current time to the End of Life (EoL). In the last several years a number of prognostic methodologies have been proposed and are mainly classified in three primary categories: the physics-based, the data-driven and the hybrid approaches. In physics-based direction, extensive knowledge about the failure mechanism is required and an explicit mathematical model has to be built for the characterization of the degradation behaviour. A spall propagation model has been proposed in [3] and is based on the spall size estimation using a vibration and debris sensor. Moreover Paris Law is another typical physical model, indicating the rate of crack growth and has been widely studied in the past. However, a real accurate physic model, representing fully the material, the lubrication, the load and the geometry properties, is hard and expensive to be developed. Moreover, the configuration for a specific type of component, i.e. a bearing is not always easily transferable to other types of the same component.

Therefore, the flexible data-driven methods have been become popular recently, deriving an analytic model directly from experimental data, ignoring the complex physics properties. The methodologies can be roughly categorised into Machine Learning methods (ANN, Deep Learning, Support Vector Machine, Relevance Vector Machine, etc.), Filtering methods (Kalman Filter, Extended Kalman Filter, Unscented Kalman Filter, Particle Filter, etc.) and Stochastic Process methods (Gaussian, Wiener) [4]. In addition, the hybrid approaches, combining data-driven and physics models, could also be a beneficial choice in the view of reliability and accuracy. On the other hand, under the consideration of applicability, the data-driven approach is becoming the focus nowadays and the prediction performance is gradually improved.

In contrast to the other two categories, filtering based methods are efficient enough to provide accurate prediction with little size of data and are suitable for any form of process noise distribution. Therefore, Kalman Filter based methods are adopted in this paper. The Extended Kalman Filter (EKF) has been proposed for the estimation of the rolling element bearing RUL tracking as features the variance and the entropy of the Choi-Williams transformation. The EKF has demonstrated promising results in comparison to the classic Kalman Filter (CKF) [5]. Moreover, the Constraint Kalman Filter (CoKF), which is a kind of computationally efficient approach, has been realized for RUL estimation and achieved encouraging accuracy in the application over a battery failure dataset [6]. In addition, the Switching Kalman Filter (SKF) has been also studied for an expanded degradation model [7], being more adequate to describe the threshold and the evolution processes, the healthy serviceable stage, the linear degradation and the exponential degradation.

However a number of research issues are still open, proved by the limited industrial maturity of PHM methods, including: the RUL prediction for high 
nonlinear systems, the model parameter initialization, the threshold setting, the parameter tuning, the performance evaluation, etc. Therefore, the objective of this paper is the application of the Unscented Kalman Filter for the estimation of RUL of rolling element bearings and the consideration of an automated procedure for the parameter tuning. The rest of the paper is organised as follows. The theory of Kalman Filtering and its versions are summarised in Section 2. Moreover the different steps of the methodology are presented in Section 3. The experimental test rig and the data set used are briefly described in Section 4 . Furthermore the results of the application are presented in Section 5. The paper is closing with some conclusions and ideas for further research mentioned in Section 6.

\section{Theory of Kalman Filter}

$\mathrm{KF}$ is a recursive estimation of a series of measurements, which have the statistical noise with Gaussian distribution. The procedure of KF consists of a prediction and an update step. In this section, different versions of KF, such as the CKF, the EKF and the UKF will be briefly discussed.

Classic Kalman Filter As the initial version, CKF is an efficient dynamic estimation by the rule of least mean square error and is suitable for the linear process and observation model:

$$
\begin{aligned}
x_{k} & =A_{k-1} x_{k-1}+B_{k-1} u_{k-1}+w_{k}, w_{k} \sim N\left(0, Q_{k}\right) \\
z_{k} & =H_{k} x_{k}+v_{k}, v_{k} \sim N\left(0, R_{k}\right)
\end{aligned}
$$

Where, $A_{k} \sim$ the state transition matrix, $B_{k} \sim$ the input matrix, $H_{k} \sim$ the observation matrix, $w_{k} \sim$ the process noise, $v_{k} \sim$ the measurement noise.

Extended Kalman Filter In reality, many processes are nonlinear, EKF is thus derived. The EKF is firstly linearised taking into account only the first order of the Taylor series w.r.t the current prediction. After linearisation, the nonlinear system fulfils the pre-conditions of CKF. For the slightly nonlinear systems, EKF achieves better prediction performance compared to the CKF. However, the ignored higher orders in the Taylor series result to an approximation error, to difficulty in estimating the Jacobian matrix in the highly nonlinear case, to non-differentiable functions and to heavy computation load.

Unscented Kalman Filter In order to overcome the limitations of EKF in the case of highly nonlinear systems, such as the negligence of higher orders of Taylor series, the UKF has been presented based on the principle of the Unscented Transform and generates a small number of sigmal points to approximate the priori and the posteriori probability distribution. Therefore, there is not necessity to calculate the Jacobian matrix in each step. In the prediction step, a number of sigma points and the corresponding weights are priori generated with a specific rule. Next, the sigma points propagate through the nonlinear function $f$ and combined with the weights. Then the predicted state and the covariance can 
be calculated. The procedure of the update step is similar to the prediction one. A new set of sigma points is generated using the predicted state through a projection over the observation function $h$. Afterwards, the posteriori state and covariance are updated with the new measurement.

\section{Methodology of the RUL prediction}

In this paper a data driven methodology for the estimation of a component's RUL is proposed based on the exploitation of training data and the Unscented Kalman Filter. A classical bottleneck of Kalman filter approaches is the need for manual tuning of a number of parameters. A particular approach has been considered here focusing towards the automated parameter tuning. The methodology can be summarised as follows. First a prognostic feature is extracted from training data. Ideally the feature should follow the component degradation presenting a monotonic trend. The training data consist of run-to-failure data sets including signals captured at constant intervals. A specific crucial selection is initially done, setting the threshold which corresponds to the EoL of the component. Moreover an appropriate parametric model, e.g. an exponential model, is selected. The model is curve-fitted on each degradation set of measurements and its parameters are extracted for each set. The model with initial parameters equal to the mean value of the parameters, extracted from the training data, is used as the model for the Kalman Filters. Additionally the threshold for the testing data is set equal to the mean value of the training thresholds. Furthermore the testing data sets are used in order to extract the selected feature and for each input signal the RUL is estimated.

The RUL is calculated through an extrapolation till the threshold $R U L_{k}=$ $t_{\text {extrap }}-t_{k}$, where $t_{\text {extrap }}$ and $t_{k}$ are respectively the predicted EoL and the current time. The $t_{\text {extrap }}$ is highly related with the sensitivity of the model parameters. Therefore the dynamic (non steady) variance w.r.t. the parameter uncertainty is studied here. Theoretically the parameters of KF stabilize around the constant $p_{s}$, if the model of the process matches well with the true process. The variance of the parameters $Q_{p}, p=\left[p_{1}, \ldots, p_{j}\right], j$ is the number of the model parameters, can be approximated by trial and error. However, the manual tuning requests time and expertise. Additionally a new tuning would be requested if a machine condition changes. Therefore it is proposed that the parameter variance is automatically tuned and it varies dynamically at each step.

The proposed parameter tuning is based on linearization and has been inspired from [8]. The original state space formula is written as the equation (3):

$$
\dot{x}=f(x, p)
$$

Where, $p \sim$ model parameters. Under the assumption of a correct model, the state constructed by the stabilized parameters $p_{s}$ is given as the expression (4):

$$
\dot{x_{s}}=f\left(x_{s}, p_{s}\right)
$$


Therefore, in the assuption of $x \approx x_{s}$ the equation (4) is rewritten as below:

$$
\dot{x}=f\left(x, p_{s}\right)+w
$$

The Taylor series w.r.t $x_{s}$ and $p_{s}$ can be written as the equation (6):

$$
\begin{aligned}
f(x, p) & =f\left(x_{s}, p_{s}\right)+\frac{\partial f}{\partial x_{x \approx x_{s}}}\left(x-x_{s}\right)+\frac{\partial f}{\partial p_{p \approx p_{s}}}\left(p-p_{s}\right)+\ldots \\
& =f\left(x_{s}, p_{s}\right)+J_{x \mid x \approx x_{s}}\left(x-x_{s}\right)+J_{p \mid p \approx p_{s}}\left(p-p_{s}\right)+\ldots
\end{aligned}
$$

Ignoring the higher orders above the first one, the noise part $w$ is expressed as:

$$
\begin{aligned}
w & =f(x, p)-f\left(x_{s}, p_{s}\right) \\
& =J_{x \mid x \approx x_{s}}\left(x-x_{s}\right)+J_{p \mid p \approx p_{s}}\left(p-p_{s}\right) \\
& =J_{p} \cdot \triangle p
\end{aligned}
$$

The formulation indicates that the uncertainty of the state $x$ is mainly caused by the variance $Q_{p}$. However, the hurdle is to achieve $p_{s}$ because of the untractable posterior distribution in the beginning. Nevertheless, the property that, the predicted parameter $p_{k}$ converges to the $p_{s}$ with increasing steps of propagation, means that the fluctuation of the $p_{k}$ will generally get smaller and equal to $p_{s}$ after infinite time. In this paper, the $p_{s}$ at the $\mathrm{k}$ step is approximated as equation (8) by the $p_{k-1}$. In fact, this approximation is the variance at the $k-1$ step. For the sake of simplicity and feasibility, the approximated variance at $k-1$ is considered as the variance at $k$.

$$
\begin{aligned}
C_{p} & =\left(p_{k}-p_{s}\right)^{2} \\
& \approx\left(p_{k}-p_{k-1}\right)^{2}
\end{aligned}
$$

Moreover the starting prediction point (SPP) is a critical factor in RUL prediction. More specifically in the case of rolling element bearings, the degradation evolves in different phases and with different rates, therefore the choice of the type and the parameters of the model can significantly vary based on the SPP. The influence of the SPP in the RUL prediction is also analysed in this paper.

Furthermore in order to evaluate and compare the performance of different methodologies in function of the selected model, the KF version, the parameter tuning, the SPP etc., 5 typical criteria at Table 1 have been considered (presenting a perfect score ps) [9], that is, accuracy (AC), mean squared error (MSE), prognostics horizon (PH), , precision (PR) and Error (ERR).

The AC describes the relative error; The MSE indicates the deviation between the prediction and actual value. $\mathrm{PH}$ counts the number of prediction points falling within the confidence interval; PR signifies the dispersion of the prediction error. ERR checks the convergence of the prediction. The achieved performance value is finally compared with the perfect score. The symbols $R e$ and $\operatorname{Pr}$ denote the actual and the predicted RUL respectively. $\mathrm{N}$ is the length of the test data, error is the difference between the actual and the predicted RUL, the coefficient $\alpha \in[1,2]$ and the confidence interval $\alpha_{c} \in[0,1]$. 
Table 1: Performance metrics

\begin{tabular}{lcc}
\hline Metrics & Equation & Perfect score $(\mathrm{ps})$ \\
\hline $\mathrm{AC}$ & $\frac{1}{N} \sum_{i=1}^{N} e^{-\frac{|\operatorname{lerror}(i)|^{a}}{R e(i)}}$ & 1 \\
$\mathrm{MSE}$ & $\frac{1}{N} \sum_{i=1}^{N} \operatorname{error}(i)^{2}$ & 0 \\
$\mathrm{PH}$ & $\forall \operatorname{Pr}(i) \in\left[\operatorname{Re}(i) \cdot\left(1-\alpha_{c}\right), \operatorname{Re}(i) \cdot\left(1+\alpha_{c}\right)\right]$ & $\mathrm{N}$ \\
$\mathrm{PR}$ & $\sqrt{\frac{\sum_{i=1}^{N}(\operatorname{error}(i)-\overline{\operatorname{error}})}{N}}$ & 0 \\
$\mathrm{ERR}$ & $\frac{|100 \cdot \operatorname{error}(N)|}{\operatorname{Re}(N)}$ & 0 \\
\hline
\end{tabular}

\section{Experimental test rig and datasets}

The methodologies are tested and evaluated on the well known experimental data of the PRONOSTIA platform [10]. The data has been captured under three operating conditions with constant speed and load (condition 1: $1800 \mathrm{rpm}$, $4000 \mathrm{~N}$; condition 2: $1650 \mathrm{rpm}, 4200 \mathrm{~N}$; condition 3: $1500 \mathrm{rpm}, 5000 \mathrm{~N})$. The setup consists of a motor, a custom gearbox, two oversized bearings and the test bearing which is loaded by a pneumatic actuator. Data is acquired each 10 seconds with a sampling frequency $f_{s}$ equal to $25.6 \mathrm{kHz}$ and a period $t$ equal to 0.1 seconds. Acceleration signals are collected simultaneously at the horizontal and the vertical direction and the measurements stop when the acceleration amplitude at either of the two directions exceeds $20 \mathrm{~g}$, which is therefore defined as the EoL. Seventeen bearings of the same type have been tested. Two data sets are used as training data for each of the three conditions while eleven truncated data sets are used for testing (Cond.1: 5, Cond.2: 5, Cond.3: 1).

\section{Results and Discussion}

The above-mentioned methodology has been applied over the PRONOSTIA measurements. As mentioned before, the duration of each signal is 0.1 seconds and the frequency resolution is very poor equal to $10 \mathrm{~Hz}$. Therefore advanced monitoring features cannot be used and as a result the statistical indicator RMS is considered in this paper.

An exponential model is selected for the modelling of the training data. A curve is fitted over the feature curve as equation (9) for the training dataset extracting the corresponding parameters $a_{k 11}, a_{k 12}, \ldots, a_{k 1 N}$ and $b_{k 11}, b_{k 12}, \ldots, b_{k 1 N}$.

$$
f e a t(k 1 i)=a_{k 1 i} \cdot e^{b_{k 1 i} \cdot k}, i=1 \ldots N
$$

The initial parameter $a_{0}, b_{0}$ and the threshold are obtained in the manner:

$$
\begin{aligned}
a_{0} & =\frac{\sum_{i=1}^{N} a_{k 1 i}}{N} \\
b_{0} & =\frac{\sum_{i=1}^{N} b_{k 1 i}}{N} \\
\text { Threshold } & =\frac{\sum_{i=1}^{N} \text { feat }_{k 1 i}(\text { end })}{N}
\end{aligned}
$$


Generally, the RUL has been estimated starting from 5 different SPPs $(0,1 / 4$, $1 / 2,3 / 4$ and $9 / 10$ of test data length) in order to investigate the influence of the SPP in the different degradation stages and the performance of the KF versions. The comparison is realised starting from the same initial parameters. Based on the lack of space, the results of the analysis are briefly demonstrated only for the SPP4 and the test data 135, which corresponds to the 3rd test data in operation condition 1 measured in the horizontal direction. The RMS value and the corresponding RUL are predicted using the CKF, EKF and UKF and are presented in Figure 1. The corresponding annotation in the legend signifies the selected feature (RMS), the reference (actual RUL) with an upper and a lower $20 \%$ CI (confidence interval), the version of $\mathrm{KF}(\mathrm{U} / \mathrm{E} / \mathrm{CKF}$ ) and the manual/automated parameter tuning.
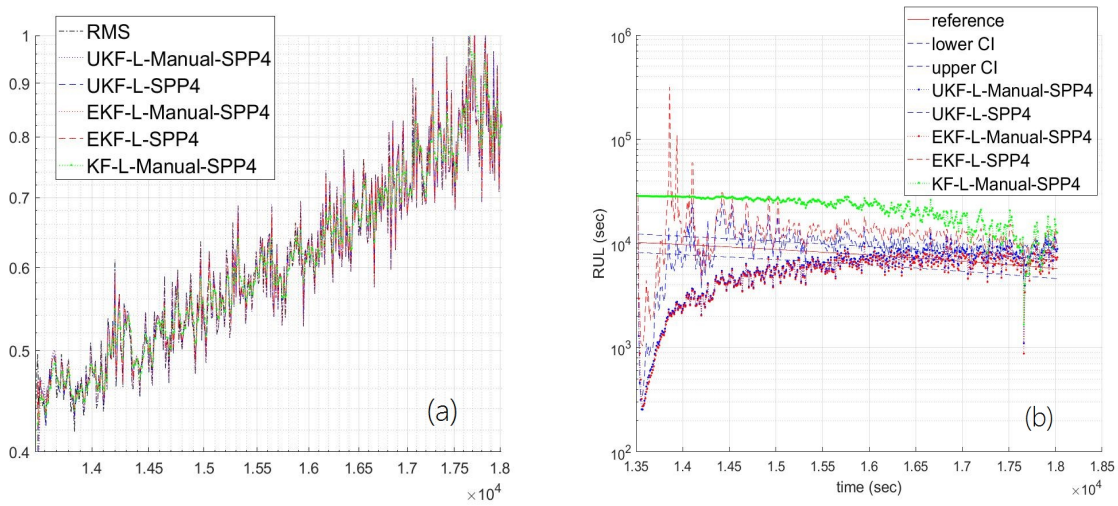

Fig. 1: Comparison of Kalman Filter versions: (a) Prediction and true RMS, (b) Prediction and actual RUL.

Analysing the Figure 1a, the UKF and the EKF based prediction coincides almost with the actual RMS and is difficult to be distinguished. On the other hand there is an obvious difference for the CKF. The corresponding RUL estimation is presented in Figure 1b. The performance of the UKF and the EKF by manual parameter tuning is equivalent with each other. An extended error occurs in the beginning but a fast convergence rate is mentioned before $14000 \mathrm{~s}$. Then the prediction approaches the reference line at around $15500 \mathrm{~s}$ but later it diverges again with a stable slope. An extended error can be identified at $17600 \mathrm{~s}$, which is directly caused by an abnormal feature point. The outliers seem to affect heavily the RUL prediction performance. From late stage performance, EKF seems to be slightly better than UKF. However, using the proposed automated parameter tuning, the results of the UKF and the EKF seem comparable.

The prediction before $14000 \mathrm{~s}$ seems to be worse than the manual tuning but it comes closer to the actual RUL. A close convergence occurs earlier, at 14200s, and then the performance remains stable till the end. The performance of the UKF is superior to the EKF and appears to be more reliable than the manual 
EKF. In addition, the CKF presents an extended error from the beginning till the end for the prediction of both the RUL and the feature value.

Apart from the visual inspection in Figure 1, the quality of the performance is also quantitatively evaluated at Table 2 with the proposed performance metrics (as shown at Table 1). The analysis of the metrics leads to the similar conclusions. In the case of manual parameter tuning, the EKF based prediction demonstrates a better performance in comparison to UKF, while the worst result is achieved by the CKF, presenting a considerable error. In the case of automated tuning, the UKF presents a better result compared to the EKF based on almost all criteria. The performance of the UKF-LR (automated version) is lower than the EKF-Manual, but in a comparable sense. Moreover the PR of the UKF-LR is better than the EKF-Manual as it demonstrates better prediction performance of the deviation dispersion for the whole duration.

Table 2: Performance metrics in case of SPP4: Test data 135

\begin{tabular}{|c|c|c|c|c|c|c|}
\hline & UKF_Manual & EKF_Manual & KF_Manual & UKF_LR & EKF_LR & Perfect score \\
\hline $\mathrm{AC}$ & $7,20 \mathrm{E}-01$ & $8,28 \mathrm{E}-01$ & $1,86 \mathrm{E}-04$ & 6,60E-01 & $5,42 \mathrm{E}-01$ & 1 \\
\hline MSE & $6,37 \mathrm{E}+06$ & $2,49 \mathrm{E}+06$ & $2,68 \mathrm{E}+09$ & $8,31 \mathrm{E}+06$ & $1,98 \mathrm{E}+07$ & 0 \\
\hline $\mathrm{PH}$ & $1,50 \mathrm{E}+01$ & $3,10 \mathrm{E}+01$ & $0,00 \mathrm{E}+00$ & $8,00 \mathrm{E}+00$ & $4,00 \mathrm{E}+00$ & 50 \\
\hline PR & $1,78 \mathrm{E}+03$ & $1,46 \mathrm{E}+03$ & $1,81 \mathrm{E}+03$ & $1,39 \mathrm{E}+03$ & $1,99 \mathrm{E}+03$ & 0 \\
\hline ERR & $-5,45 \mathrm{E}+01$ & $-2,83 \mathrm{E}+01$ & $-9,10 \mathrm{E}+02$ & $-5,57 \mathrm{E}+01$ & $-7,85 \mathrm{E}+01$ & 0 \\
\hline
\end{tabular}

Details over the other four SPPs (SPP1,SPP2,SPP3,SPP5) for dataset 135 are not presented due to the lack of space. Briefly, in the case of SPP1, it's noticeable that the UKF-LR provides better results compared to the manual EKF and the CKF, but achieves a lower performance compared to the UKF-Manual. A similar performance is achieved in the case of SPP2. The UKF in combination with the automated tuning indicates better prediction compared to the UKF and the EKF using manual parameter tuning. For SPP3, the UKF-LR indicates superiority as in the case of SPP2. However, when the prediction point starts close to the final stage of life like the SPP5, the EKF-LR shows a better outcome compared to the manual UKF and EKF. Therefore, in general view, the algorithm of UKF-LR gives out the best prediction for the test data 135. In the same manner, the best prediction for the test data 145, 155, 165, 175 are always released by the UKF-LR. The results of the prediction for all the test datasets are summarized at Table 3.

Table 3: RUL prediction results

\begin{tabular}{lccc}
\hline Operating condition & Condition $(\mathrm{X}=1)$ & Condition $(\mathrm{X}=2)$ & Condition $(\mathrm{X}=3)$ \\
\hline Testdata $(\mathrm{X}) 35$ & UKF-LR & EKF-LR & EKF-LR \\
Testdata (X) 45 & UKF-LR & UKF-LR & \\
Testdata $(\mathrm{X}) 55$ & UKF-LR & UKF-LR & \\
Testdata $(\mathrm{X}) 65$ & UKF-LR & UKF-LR & \\
Testdata $(\mathrm{X}) 75$ & UKF-LR & UKF-LR & \\
\hline
\end{tabular}

The UKF is acknowledged as the favourable choice in the first two operation 
conditions, with high speed and lower radial load, with the exception of the test data 235. Under the condition of heavy load and lower speed, the EKF is seemingly more prominent than the UKF, but as mentioned before there is only one single test data in condition 3.

Finally, the dynamic variation of the parameters $a$ and $b$, corresponding to the performance of Figure 1 is presented in Figure 2. The constant variance $Q_{a}$ and $Q_{b}$ for the EKF and the UKF are initially set equal to $10^{-6}$ by experience. The time-varying variance $Q_{a}$ is demonstrated in Figure 2a. A large variance is identified at the beginning and a fluctuation is present which generally is damped with time. The dynamic value of the EKF seems to be slightly lower than the UKF. The variance of the parameter $b$ is shown in Figure $2 \mathrm{~b}$. Around the $143000 s$, the variance of the UKF and the EKF goes below $10^{-6}$, which explains the fast convergence identified at the prediction performance in Figure 1. Additionally the variances $Q_{a}$ and $Q_{b}$ are both lower at the EKF compared to the UKF.
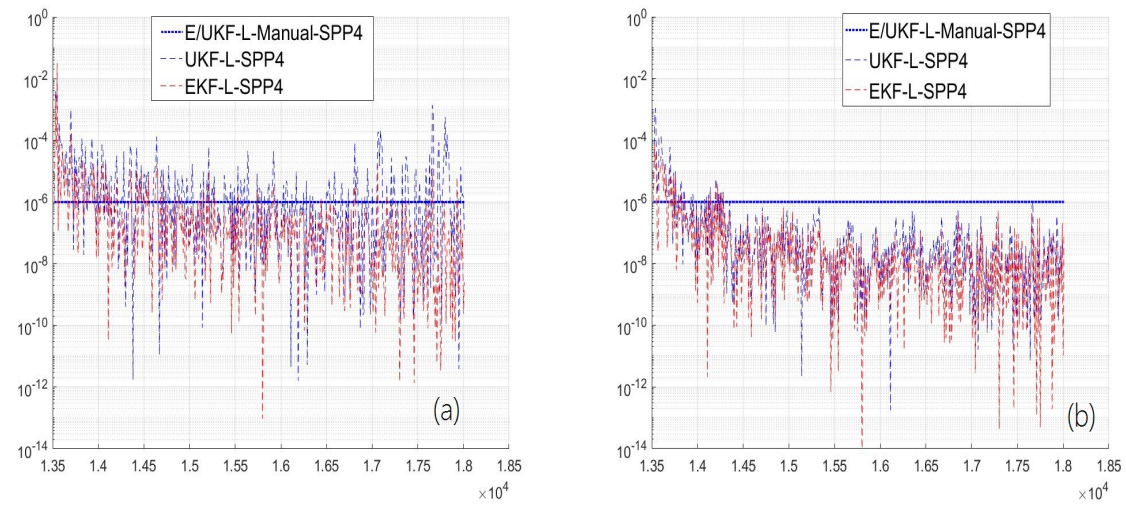

Fig. 2: Parameter variation: (a) Variance propagation of parameter a, (b) Variance propagation of parameter $b$

\section{Conclusion}

In this paper, the Kalman filter based RUL prediction is studied and existing challenges are highlighted and investigated, including the parameters initialization, the threshold setting, the start prediction point, the performance evaluation, etc. Different types of Kalman Filter have been considered and combined with an automatic parameter tuning methodology. Through comparison using the PROGNOSTIA experimental data, the Unscented Kalman Filter yields in general better performance compared to the classic and the Extended Kalman Filter. Moreover the influence of the start prediction point to the RUL prediction has been investigated. A start time between SPP4 and SPP5 is recommended as the convergence towards the actual RUL during this interval is closer compared to other starting points. It is not advisable to start the prediction after the SPP5, as the bearings go to failure very soon. Additionally, 
a solution to the classical problem of parameter tuning has been proposed. The automated parameter tuning delivers an adaptive covariance and performs in combination with the EKF and the UKF better than the manual tuning based on trial and error expertise. As extension of this work, the authors are working on the application of the methodologies on different data sets using more advanced monitoring features as well as on the optimisation of different parts of the methodology including the detection of feature outliers, the improvement and optimization of the proposed parameter tuning, the automatically recognition of the optimal start point, the use of an adaptive model etc.

\section{Acknowledgment}

K. Gryllias would like to gratefully acknowledge the Research Fund KU Leuven.

\section{References}

1. Optimizing Operations and Maintenance with Predictive Analytics. https://blog.schneider-electric.com/industrial-software/ 2015/07/17/optimizing-operations-maintenance-predictive-analytics /

2. Lee, J., Jin, C., Liu, Z.C., Ardakani H.D.: Introduction to Data-Driven Methodologies for Prognostics and Health Management. Probabilistic Prognostics and Health Management of Energy Systems, Springer International Publishing AG 2017. doi:10.1007/978-3-319-55852-3_2

3. Bolander, N., Qiu, H., Eklund, N., Hindle, E., Rosenfeld T.: Physics-based Remaining Useful Life Prediction for Aircraft Engine Bearing Prognosis. In: Annual Conference of the Prognostics and Health Management Society (2009).

4. Wu, L.F., Fu, X.H., Guan, Y.: Review of the Remaining Useful Life Prognostics of Vehicle Lithium-Ion Batteries Using Data-Driven Methodologies. Applied sciences, Volume 6, Issue 6, pp. 1-11 (2016). doi:10.3390/app6060166

5. Singleton, R.K., Strangas, E.G., Aviyente, S.: Extended Kalman Filtering for Remaining-Useful-Life Estimation of Bearings. IEEE Transactions on Industrial Electronics, Volume: 62, Issue 3 (2015). doi:10.1109/TIE.2014.2336616

6. Son, J.B., Zhou, S.Y., Sankavaram, C., Du, X.Y., Zhang, Y.L.: Remaining useful life prediction based on noisy condition monitoring signals using constrained Kalman filter. Reliability Engineering System Safety, Volume 152, pp. 38-50 (2016). doi: 10.1016/j.ress.2016.02.006

7. Lim, R., Mba, D.: Condition Monitoring and Remaining Useful Life Prediction using Switching Kalman Filter. Int. J. Strategic Engineering Asset Management, Vol. 2, No. 1 (2014). doi:10.1504/IJSEAM.2014.063881

8. Valappil, J., Georgakis, C.: A Systematic Tuning Approach for the Use of Extended Kalman Filters in Batch Processes. In: Proceedings of the Arnencan Control Conference, San Diego, California (1999). doi:10.1109/ACC.1999.783220

9. Saxena, A., Celaya, J., Saha, B., Saha, S., Goebel, K.: Metrics for Offline Evaluation of Prognostic Performance. International Journal of Prognostics and Health Management (2010).

10. Nectoux, P., Gouriveau, R., Medjaher, K., Ramasso, E., Chebel- Morello, B.: PRONOSTIA: An experimental platform for bearings accelerated degradation tests. In: IEEE International Conference on Prognostics and Health Management, PHM'12., Denver, Colorado, United States (2012). 